PROCEEDINGS OF THE

AMERICAN MATHEMATICAL SOCIETY

Volume 125, Number 4, April 1997, Pages 1215-1220

S $0002-9939(97) 03686-1$

\title{
THE STRUCTURE OF FUNCTIONS SATISFYING THE LAW OF LARGE NUMBERS IN A CLASS OF LOCALLY CONVEX SPACES
}

\author{
ROBERT C. STOLZ
}

(Communicated by Richard T. Durrett)

\begin{abstract}
For each function $f$ that satisfies the law of large numbers with values in a certain class of locally convex spaces with the Radon-Nikodym property the following decomposition holds: $f=f_{1}+f_{2}$, where $f_{1}$ is integrable by seminorm, and $f_{2}$ is a Pettis integrable function which is scalarly 0 .
\end{abstract}

\section{INTRODUCTION}

In this paper, $(\Omega, \Sigma, \mu)$ denotes a probability space, $(F, \mathcal{P})$ a complete locally convex space, where $\mathcal{P}$ is a generating family of seminorms, and $F^{\prime}$ the topological dual of $F$. If the topology on $F$ is generated by a single seminorm $q$, then this space will be denoted by $(F, q)$. Let

$$
U_{q}=\{x \in F \mid q(x) \leq 1\} \text { and } U_{q}^{\circ}=\left\{x^{\prime} \in F^{\prime}|| x^{\prime} y \mid \leq 1, \forall y \in U_{q}\right\} .
$$

For every $f \in F^{\Omega}$, denote:

$$
\begin{aligned}
q_{P}(f) & =\sup _{x^{\prime} \in U_{q}^{\circ}} \int_{\Omega}^{*}\left|x^{\prime} f\right| d \mu, \\
q_{G C}(f) & =\limsup _{n \rightarrow \infty} \int_{\Omega^{\infty}}^{*} \sup _{x^{\prime} \in U_{q}^{\circ}} \frac{1}{n} \sum_{i=1}^{n}\left|x^{\prime} f\left(\xi_{i}\right)\right| d \mu^{\infty}\left(\left(\xi_{i}\right)\right), \\
q_{1}(f) & =\int_{\Omega}^{*} q(f) d \mu .
\end{aligned}
$$

Set

$$
\begin{aligned}
\operatorname{LLN}(\mu, F)= & \{f: \Omega \rightarrow F \mid \exists a \in F: \\
& \left.\left(\mu^{\infty}\right)_{*}\left\{\left(\omega_{i}\right) \in \Omega^{\infty} \mid \lim _{n \rightarrow \infty} q\left(\frac{1}{n} \sum_{i=1}^{n} f\left(\omega_{i}\right)-a\right)=0, \forall q \in \mathcal{P}\right\}=1\right\},
\end{aligned}
$$

Received by the editors July 14, 1995 and, in revised form, October 10, 1995.

1991 Mathematics Subject Classification. Primary 60B12.

Key words and phrases. The law of large numbers, locally convex spaces.

The present paper is part of the author's doctoral thesis and was carried out under the supervision of Professor V. Dobrić during a stay at Lehigh University.

(C)1997 American Mathematical Society 


$$
\begin{aligned}
\mathcal{M}(\Sigma, \mathcal{F}) & =\{f: \Omega \rightarrow F \mid f \text { is }(\Sigma, \mathcal{F}) \text {-measurable }\}, \\
L_{P}^{1}(\mu, F) & =\{f: \Omega \rightarrow F \mid f \text { is Pettis } \mu \text {-integrable }\}, \\
L_{*}^{1}(\mu, F) & =\left\{f: \Omega \rightarrow F \mid q_{1}(f)<\infty \forall q \in \mathcal{P}\right\}, \\
L_{S}^{1}(\mu, F) & =\{f: \Omega \rightarrow F \mid f \text { is } \mu \text {-integrable by seminorm }\},
\end{aligned}
$$

where a function $f: \Omega \rightarrow F$ is said to be $\mu$-integrable by seminorm [2] if for each $q \in \mathcal{P}$ there exist a set $N_{q}$ with $\mu\left(N_{q}\right)=0$ and a sequence $\left\{s_{n}^{q}\right\}$ of simple functions such that:

1. $\lim _{n \rightarrow \infty} q\left(f(\omega)-s_{n}^{q}(\omega)\right)=0$ for each $\omega \in \Omega \backslash N_{q}$, i.e. $f$ is measurable by seminorm;

2. $q\left(f(\omega)-s_{n}^{q}(\omega)\right) \in L_{1}(\mu, \mathbf{R})$ for every $n \in \mathbf{N}$ and

$$
\lim _{n \rightarrow \infty} \int_{\Omega} q\left(f-s_{n}^{q}\right) d \mu=0
$$

3. for each $A \in \Sigma$, there exists $y_{A} \in F$ such that for every $q \in \mathcal{P}$

$$
\lim _{n \rightarrow \infty} q\left(\int_{A} s_{n}^{q} d \mu-y_{A}\right)=0 .
$$

We then define $y_{A}=\int_{A} f d \mu$. Integrability by seminorm, first introduced by Grothendieck [7], is one of the possible extensions of Bochner integrability from Banach to locally convex spaces.

V. Dobrić [5] has shown that for a locally convex space $F$

$$
L L N(\mu, F) \subseteq L_{P}^{1}(\mu, F) \cap L_{*}^{1}(\mu, F) .
$$

E. Mourier [11] proved for separable Banach spaces and A. Beck [1] for nonseparable Banach spaces that the set of functions that are Bochner integrable is contained in the set of functions that satisfy the law of large numbers.

The following section will demonstrate the inclusion for locally convex spaces,

$$
L_{S}^{1}(\mu, F) \subseteq L L N(\mu, F),
$$

provided that one of the following conditions holds:

(a) There exists a map $\Psi: \mathbf{N}^{\mathbf{N}} \rightarrow \mathcal{P}$ which is increasing, cofinal and for every $x \in F, \sigma \mapsto \Psi(\sigma)(x)$ is continuous. We will say that a locally convex space $F$ is of $\mathbf{N}^{\mathbf{N}}$-continuous type if it has this property.

(b) $F$ is an $\mathcal{F}$-analytically normed space [4] and $f \in \mathcal{M}(\Sigma, \mathcal{F})$.

Remark 1. If (a) holds, then $F$ is $\mathcal{B}(F)$-analytically normed, but we do not need to place a measurability condition to get the inclusion.

Whenever

$$
L_{S}^{1}(\mu, F) \subseteq L L N(\mu, F) \subseteq L_{P}^{1}(\mu, F) \cap L_{*}^{1}(\mu, F),
$$

it is natural to ask how far from $L_{S}^{1}(\mu, F)$ and how close to $L_{P}^{1}(\mu, F)$ the set $L L N(\mu, F)$ can be. A locally convex space $F$ is said to possess the Radon-Nikodym property if for every finite measure space $(\Omega, \Sigma, \mu)$, every $\mu$-continuous $F$-valued measure with bounded variation has a density which is integrable by seminorm. For the locally convex spaces with the Radon-Nikodym property that have property (a) or (b) the following sections will present a decomposition theorem which addresses this question. 
The general method used will obtain the results by establishing the decomposition for every seminormed space first and then transferring these results to a locally convex space. In general locally convex spaces, the transfer of almost sure results from each seminormed space to a locally convex space requires determining when a union of null sets, each arising from the space $(F, q), q \in \mathcal{P}$, is a null set. If $\mathcal{P}$ is uncountable, such a union does not have to be a null set and additional conditions on $\mathcal{P}$ are needed. One can ensure that this uncountable union of null sets is a null set through the use of a monotone convergence theorem for nets. Conditions (a) or (b) are sufficient for the monotone convergence theorem for nets developed by Hoffmann-Jørgensen [8] to hold.

\section{Decomposition Theorem}

The following result gives conditions under which almost sure convergence in $F$ is equivalent to almost sure convergence in $(F, q)$ for all $q \in \mathcal{P}$.

Theorem 1. Let $f_{n}$ be a sequence of functions such that $q f_{n} \rightarrow 0 \mu$-a.s. for every $q \in \mathcal{P}$ and $q f_{n}$ is $\left(\Sigma, \mathcal{B}(\mathbf{R})\right.$ )-measurable for every $q \in \mathcal{P}$. If $F$ is of $\mathbf{N}^{\mathbf{N}}$-continuous type, then $f_{n} \rightarrow 0$ in $F \mu$-a.s.

Proof. Let $\Psi(\sigma)=q_{\sigma}$ and

$$
N_{q_{\sigma}}=\left\{\omega \in \Omega: q_{\sigma}\left(f_{n}(\omega)\right) \nrightarrow 0\right\} .
$$

If we show that

$$
M=\bigcup_{\sigma \in \mathbf{N}^{\mathbf{N}}} N_{q_{\sigma}} \text { is a null set, }
$$

the proof will be complete. The following is in the same spirit as V. Dobrić's work on analytically normed spaces [4, Theorem 3.3].

Let $\Theta=\left\{1_{N_{q}}: q \in \mathcal{P}\right\}$. If we prove that $\Theta$ is smoothly filtering upwards on $(\Omega, \Sigma, \mu)$ (see [4]), then, since $\mu^{*}\left(N_{q}\right)=0$, or $\mu\left(N_{q}\right)=0$ if $(\Omega, \Sigma, \mu)$ is complete, $\forall q \in \mathcal{P}$, by [8, Corollary 2.3], $\mu^{*}(M)=0$.

Let $\Phi: \mathbf{N}^{\mathbf{N}} \rightarrow\left\{1_{N_{q}}: q \in \mathcal{P}\right\}$ be defined by $\Phi(\sigma)=1_{N_{q_{\sigma}}}$. $\Phi$ is increasing since $N_{q_{\sigma}} \subseteq N_{q_{\tau}}$ if $\sigma \leq \tau$. $\Phi$ is cofinal since the map $\Psi$ is cofinal. It remains to prove

$$
S_{a}=\left\{(\sigma, \omega) \in \mathbf{N}^{\mathbf{N}} \times \Omega: \Phi(\sigma)(\omega)>a\right\} \in \mathbf{S}\left(\mathcal{B}\left(\mathbf{N}^{\mathbf{N}}\right) \otimes \Sigma\right) \quad \forall a>0 .
$$

If $a>1$, then $S_{a}=\emptyset \in \mathbf{S}\left(\mathcal{B}\left(\mathbf{N}^{\mathbf{N}}\right) \otimes \Sigma\right)$. If $0<a \leq 1$, then

$$
\begin{aligned}
S_{a} & =\left\{(\sigma, \omega): \omega \in N_{q_{\sigma}}\right\} \\
& =\left\{(\sigma, \omega): q_{\sigma}\left(f_{n}(\omega)\right) \nrightarrow 0\right\} \\
& =\bigcup_{n=1}^{\infty} \bigcap_{m=1}^{\infty} \bigcup_{k=m}^{\infty}\left\{(\sigma, \omega): q_{\sigma}\left(f_{k}(\omega)\right) \geq \frac{1}{n}\right\} .
\end{aligned}
$$

Since Souslin sets are closed under countable intersections and unions [12], one needs only to show

$$
\left\{(\sigma, \omega): q_{\sigma}\left(f_{k}(\omega)\right) \geq \frac{1}{n}\right\} \in \mathbf{S}\left(\mathcal{B}\left(\mathbf{N}^{\mathbf{N}}\right) \otimes \Sigma\right) .
$$

Let us define $h_{n}: \mathbf{N}^{\mathbf{N}} \times \Omega \rightarrow \mathbf{R}$ by $h_{n}(\omega, \sigma)=q_{\sigma}\left(f_{n}(\omega)\right)$. Hypotheses assure that $h_{n}(\cdot, \omega)$ is continuous for every $\omega \in \Omega$ and $h_{n}(\sigma, \cdot)$ is $(\Sigma, \mathcal{B}(\mathbf{R}))$-measurable for every 
$\sigma \in \mathbf{N}^{\mathbf{N}}$. Since $\mathbf{N}^{\mathbf{N}}$ admits $\mathcal{B}\left(\mathbf{N}^{\mathbf{N}}\right)$-elementary resolution of the identity [9], we get that $h_{n}$ is $\left(\mathcal{B}\left(\mathbf{N}^{\mathbf{N}}\right) \otimes \Sigma, \mathcal{B}(\mathbf{R})\right)$-measurable (see [9, Theorem IV.2.6]). It follows that

$$
\left\{(\sigma, \omega): q_{\sigma}\left(f_{k}(\omega)\right) \geq \frac{1}{n}\right\} \in \mathcal{B}\left(\mathbf{N}^{\mathbf{N}}\right) \otimes \Sigma .
$$

Thus, (2.1) holds.

Corollary 1. Let $S_{n}\left(\left(\omega_{i}\right)\right)=a-\frac{1}{n} \sum_{i=1}^{n} f\left(\omega_{i}\right)$. Suppose

$$
\left(\mu^{\infty}\right)_{*}\left\{\left(\omega_{i}\right) \in \Omega^{\infty}: \lim _{n} q\left(S_{n}\left(\left(\omega_{i}\right)\right)\right)=0\right\}=1 \forall q \in \mathcal{P}
$$

and $q S_{n}$ is $\left(\Sigma^{\infty}, \mathcal{B}(\mathbf{R})\right)$-measurable. If $F$ is of $\mathbf{N}^{\mathbf{N}}$-continuous type, then

$$
\left(\mu^{\infty}\right)_{*}\left\{\left(\omega_{i}\right) \in \Omega^{\infty}: \lim _{n} q\left(S_{n}\left(\omega_{i}\right)\right)=0 \quad \forall q \in \mathcal{P}\right\}=1
$$

Proof. Let $f_{n}\left(\left(\omega_{i}\right)\right)=S_{n}\left(\left(\omega_{i}\right)\right)$ and apply Theorem 1 .

Using the above corollary we can establish the following proposition.

Proposition 1. If $F$ is of $N^{N}$-continuous type, then

$$
L_{S}^{1}(\mu, F) \subseteq L L N(\mu, F)
$$

Proof. If $f \in L_{S}^{1}(\mu, F)$, then $f \in L^{1}(\mu,(F, q))$ for every $q \in \mathcal{P}$. It follows from [10, Theorem 2.4] and [5, Lemma 2.4] that $f \in L L N(\mu,(F, q))$ for every $q$ in $\mathcal{P}$ and $q S_{n}$ is $\left(\Sigma^{\infty}, \mathcal{B}(\mathbf{R})\right)$-measurable, since $f \in L^{1}(\mu,(F, q))$. A direct consequence of Corollary 1 is that $f \in L L N(\mu, F)$.

Theorem 2. Let $F$ be a locally convex space with the Radon-Nikodym property of $N^{N}$-continuous type. If $f \in L L N(\mu, F)$, then there exists $f_{1} \in L_{S}^{1}(\mu, F)$ and $f_{2} \in L_{P}^{1}(\mu, F), q_{P}\left(f_{2}\right)=0$ for each $q \in \mathcal{P}$, such that

$$
f=f_{1}+f_{2} \text {. }
$$

Moreover, the decomposition (2.2) is unique.

Conversely, if $f_{1} \in L_{S}^{1}(\mu, F)$ and $f_{2} \in L_{P}^{1}(\mu, F)$ such that $q_{G C}\left(f_{2}\right)=0$ and $q S_{n, 2}$ is $\left(\Sigma^{\infty}, \mathcal{B}(\mathbf{R})\right)$-measurable for each $q \in \mathcal{P}$, where $S_{n, 2}\left(\left(\omega_{i}\right)\right)=\frac{1}{n} \sum_{i=1}^{n} f_{2}\left(\omega_{i}\right)$, then $f_{1}+f_{2} \in L L N(\mu, F)$.

Proof. Let $f=f_{1}+f_{2}$, where $f_{1} \in L_{S}(\mu, F)$ and $f_{2} \in L_{P}^{1}(\mu, F), q_{G C}\left(f_{2}\right)=0$ for every $q \in \mathcal{P}$. Talagrand [14] proved that $g \in L L N(\mu,(F, q))$ if and only if there exists a sequence $\left\{s_{n}^{q}\right\}$ of simple functions such that

$$
q_{G C}\left(g-s_{n}^{q}\right) \rightarrow 0 \text { as } n \rightarrow \infty .
$$

Choose $s_{n}^{q}=0$ for every $n \in \mathbf{N}$. Then (2.3) holds for $f_{2}$ and so $f_{2} \in \operatorname{LLN}(\mu,(F, q))$ and $q S_{n, 2}$ is $\left(\Sigma^{\infty}, \mathcal{B}(\mathbf{R})\right)$-measurable for each $q \in \mathcal{P}$. Again, by Corollary $1, f_{2} \in$ $\operatorname{LLN}(\mu, F)$. By Proposition $1, L_{S}^{1}(\mu, F) \subseteq L L N(\mu, F)$ and $L L N(\mu, F)$ is a vector space. Thus the converse is proved.

Let $f \in L L N(\mu, F)$. Then (1.1) implies $f \in L_{P}(\mu, F)$. Construct the vectorvalued measure

$$
G(E)=(P)-\int_{E} f d \mu \text { for every } E \in \Sigma,
$$


where $P$ stands for the Pettis integral. Then $G$ is a countably additive $\mu$-continuous vector measure on $\Sigma$ (see [3]). Since

$$
f \in L L N(\mu,(F, q)) \subseteq L_{P}^{1}(\mu,(F, q)) \cap L_{*}^{1}(\mu,(F, q)),
$$

for every $q \in \mathcal{P}$ (see [5]), it follows from $[6]$ that $|G|_{q}(\Omega)<\infty$ holds for each $q \in \mathcal{P}$. Hence the vector measure $G$ is of bounded variation.

By assumption $F$ has the Radon-Nikodym property and so there exists $f_{1} \in$ $L_{S}^{1}(\mu, F)$ such that

$$
G(E)=\int_{E} f_{1} d \mu \text { for every } E \in \Sigma .
$$

For a fixed $q \in \mathcal{P}$ it follows directly from $[6]$ that $q_{P}\left(f-f_{1}\right)=0$. Set $f_{2}=f-f_{1}$. Then $f_{2} \in L L N(\mu, F)$.

To prove the decomposition (2.2) is unique, suppose there exists $g_{1} \in L_{S}^{1}(\mu, F)$ such that $q_{P}\left(f-g_{1}\right)=0$ for every $q \in \mathcal{P}$. To show uniqueness in $L_{S}(\mu, F)$ we need to prove $q\left(f_{1}-g_{1}\right)=0$ a.e. for every $q \in \mathcal{P}$.

Fix $q \in \mathcal{P}$. Let $x^{*} \in(F, q)^{\prime}$ be arbitrary. Choose $n$ such that $x^{*} \in n U_{q}^{\circ}$, which implies $\frac{x^{*}}{n} \in U_{q}^{\circ}$. Since $q_{P}\left(f_{1}-g_{1}\right)=0$,

$$
\int_{\Omega}\left|\frac{x^{*}}{n}\left(f_{1}-g_{1}\right)\right| d \mu=0
$$

which implies $x^{*} f_{1}=x^{*} g_{1} \mu$-a.e. But then, since $f_{1}, g_{1} \in L_{1}(\mu,(F, q)), q\left(f_{1}-g_{1}\right)=$ $0 \mu$-a.e. by [3, Corollary II.2.7]. Thus the proof is complete.

Example 1. Test spaces $\mathcal{D}(S)$ described in [13] give one example of a locally convex space with the Radon-Nikodym property [2], where there exists an increasing cofinal map $\Psi: \mathbf{N}^{\mathbf{N}} \rightarrow \mathcal{P}$ such that $\sigma \mapsto p_{\sigma}(x)$ is continuous for each $x \in F$ [4].

In what follows we will consider $\mathcal{F}$-analytically normed spaces [4] and develop a decomposition theorem for $(\Sigma, \mathcal{F})$-measurable functions.

Proposition 2. Let $\mathcal{F}$ be a $\sigma$-algebra on $F$ so that $\mathcal{F}$ is analytically normed. Then

$$
\mathcal{M}(\Sigma, \mathcal{F}) \cap L_{S}^{1}(\mu, F) \subseteq L L N(\mu, F) .
$$

Proof. If $f \in L_{S}^{1}(\mu, F)$, then $f \in L L N(\mu,(F, q))$ for every $q \in \mathcal{P}$. By hypothesis $f$ is $(\Sigma, \mathcal{F})$-measurable, so by [4, Corollary 3.4] $f \in L L N(\mu, F)$.

Hence, the following inclusions hold, provided $(F, \mathcal{F})$ is an $\mathcal{F}$-analytically normed space:

$$
\mathcal{M}(\Sigma, \mathcal{F}) \cap L_{S}^{1}(\mu, F) \subseteq L L N(\mu, F) \subseteq L_{P}^{1}(\mu, F) \cap L_{*}^{1}(\mu, F) .
$$

We are now in a position to prove the following decomposition theorem.

Theorem 3. Let $F$ be an $\mathcal{F}$-analytically normed locally convex space with the Radon-Nikodym property. If $f \in L L N(\mu, F)$, then there exist $f_{1} \in L_{S}^{1}(\mu, F)$ and $f_{2} \in L_{P}^{1}(\mu, F), q_{P}\left(f_{2}\right)=0$ for each $q \in \mathcal{P}$, such that

$$
f=f_{1}+f_{2} .
$$

Moreover, the decomposition (2.4) is unique.

Conversely, if $f_{1} \in L_{S}^{1}(\mu, F) \cap \mathcal{M}(\Sigma, \mathcal{F})$ and $f_{2} \in L_{P}^{1}(\mu, F) \cap \mathcal{M}(\Sigma, \mathcal{F})$ where $q_{G C}\left(f_{2}\right)=0$ for each $q \in \mathcal{P}$, then $f_{1}+f_{2} \in L L N(\mu, F)$. 
Proof. Let $f=f_{1}+f_{2}$, where $f_{1} \in L_{S}(\mu, F) \cap \mathcal{M}(e, \mathcal{F})$ and $f_{2} \in L_{P}^{1}(\mu, F) \cap \mathcal{M}(\Sigma, \mathcal{F})$, $q_{G C}\left(f_{2}\right)=0$ for every $q \in \mathcal{P}$. The same argument as in the proof of Theorem 1 assures that $f_{2} \in L L N(\mu,(F, q))$ for each $q \in \mathcal{P}$. By [4, Corollary 3.4], $f_{2} \in L L N(\mu, F)$, since $f_{2}$ is $(\Sigma, \mathcal{F})$-measurable. Because $L_{S}^{1}(\mu, F) \cap \mathcal{M}(\Sigma, \mathcal{F}) \subseteq$ $L L N(\mu, F)$ and $L L N(\mu, F)$ is a vector space, the converse is proved.

The other part of the proof is the same as the proof of Theorem 2 .

Example 2. The distribution spaces, $\mathcal{D}^{\prime}(S)$ (see [13]), are $\mathcal{B}\left(\mathcal{D}^{\prime}(S)\right.$ )-analytically normed [4] and have the Radon-Nikodym property [2]. Hence $\mathcal{D}^{\prime}(S)$ is an example of a locally convex space where this decomposition theorem holds.

\section{REFERENCES}

[1] Beck, A., On the law of large numbers, Ergodic Theory, Proc. Int. Symp. New Orleans 1961, Academic Press, 1963. MR 28:2188

[2] Blondia, C., Locally convex spaces with Radon-Nikodym property, Math. Nachr., 114 (1983), 335-341. MR 85i:46003

[3] Diestel, J. and Uhl, J.J., Vector Measures, Math. Surveys 15, American Mathematical Society, Providence, RI, 1977. MR 56:12216

[4] Dobrić, V., Analytically normed spaces, Math. Scand. 60 (1987), 109-128. MR 89c:46014

[5] _ The law of large numbers in locally convex spaces, Prob. Theor. and Related Fields 78 (1988), 403-417. MR 89e:60065

[6] _ The decomposition theorem for functions satisfying the law of large numbers, J. Theor. Prob. 3 (1990), 189-196. MR 91m:60012

[7] Grothendieck, A., Produits tensoriels topologiques et espaces nucleaires, Mem. Amer. Math. Soc. 16 (1967). MR 17:763c

[8] Hoffmann-Jørgensen, J., Stochastic Processes on Polish Spaces, Aarus Univ. Inst. Various Pub. Ser. 39, 1991. MR 95a:60047

[9] _ The Theory of Analytic spaces, Aarus Univ. Mat. Inst. Various Pub. Ser. 10, 1970. MR 53:13500

[10] — The law of large numbers for non-measurable and non-separable random elements, Asterisque 131 (1985), 299-356. MR 88a:60014

[11] Mourier, E., Elements aleatoires dans un espace de Banach, Ann. Inst. Poincare 13 (1953), 161-299. MR 16:268a

[12] Rogers, C. A. and Jayne J. E., K-analytic sets, in Analytic Sets, Academic Press, Inc., London, pp. 2-181, 1980 MR 82m:03063

[13] Rudin, W. Functional Analysis, McGraw-Hill Book Co., New York-London, 1973. MR 51:1315

[14] Talagrand, M., The Glivenko-Cantelli problem, Ann. Probab. 15 (1987), 837-870. MR 88h:60012

Department of Mathematics, Lafayette College, Easton, Pennsylvania 18042

E-mail address: StolzR@lafayette.edu

Current address: Division of Science and Mathematics, University of the Virgin Islands, St.

Thomas, Virgin Islands 00802

E-mail address: Robert.Stolz@uvi.edu 